\title{
Cent ans de musées à Taiwan, du colonialisme au nationalisme
}

Vers un imaginaire au-delà de l'identité ?

One hundred Years of Museums in Taiwan, from colonialism to nationalism:

Towards a conceptual vision that transcends identity?

\section{Lee Wei-i}

\section{OpenEdition}

\section{Journals}

Édition électronique

URL : http://journals.openedition.org/transcontinentales/668

ISBN : 978-2-7351-1565-5

ISSN : 1775-397X

\section{Éditeur}

Editions de la maison des sciences de l'homme

\section{Édition imprimée}

Date de publication : 1 juin 2007

Pagination : 145-167

ISBN : 978-2-200-92396-9

ISSN : 1950-1684

Référence électronique

Lee Wei-i, «Cent ans de musées à Taiwan, du colonialisme au nationalisme », Transcontinentales [En ligne], 4 | 2007, document 9, mis en ligne le 28 avril 2011, consulté le 07 septembre 2020. URL : http:// journals.openedition.org/transcontinentales/668 


\title{
Cent ans de musées à Taiwan, du colonialisme au nationalisme
}

\author{
Vers un imaginaire au-delà de l'identité?
}

\author{
Lee Wei-i
}

\begin{abstract}
Au terme des évolutions politiques, des compétitions pour les ressources et des conflits communautaires, les vainqueurs se sont souvent arrogé un droit d'instauration de la symbolique culturelle qui consolide la légitimité de leur domination. D'un autre côté, le recours à la symbolique culturelle peut aussi constituer un moyen de rassemblement et de cohésion pour les dominés cherchant à organiser leur résistance aux forces dominantes. Le musée, parce qu'il possède une double nature de représentation culturelle et d'instrument politique, constitue un terrain d'observation privilégié de ce phénomène.
\end{abstract}

Dans les sociétés occidentales, lors du passage du régime monarchique à celui de l'État-nation, la construction de musées nationaux a voulu conforter la domination d'une symbolique culturelle nationaliste ${ }^{1}$, alors que le communautarisme, l'autonomisation de la société civile, ainsi que la démocratisation de la culture se traduisent souvent par la construction de musées folkloriques ${ }^{2}$, locaux et ethniques pour les différents groupes et communautés qui veulent ainsi mobiliser leur identité face à la centralisation culturelle. Quant aux nouveaux États qui ont accédé à l'indépendance après la seconde guerre mondiale, le développement des musées s'y avère étroitement lié à la construction nationale postcoloniale ainsi qu'aux problèmes d'identification culturelle ${ }^{3}$.

1 - Krzystof Pomian, Collectionneurs, amateurs et curieux, Paris Venise, XVI ${ }^{e}$-XVIII ${ }^{e}$ siècle, Paris, Gallimard, 1987. Dominique Poulot, Musée, nation, patrimoine. 1789-1815, Paris, Gallimard, 1997.

2 - Anne-Marie Thiesse, La création des identités nationales : Europe XVIII ${ }^{e}-\mathrm{XX}{ }^{e}$ siècle, Paris, Seuil, 1999.

3 - Benedict Anderson, Imagined Communities. Reflections on the Origin and Spread of Nationalism, $2^{e}$ édition, Londres, Verso, 1991. Anne Gaugue, Les États africains et leurs musées. La mise en scène de la Nation, Paris, L'Harmattan, 1999. Gérard Collomb, «Ethnicité, nation, musée, en situation postcoloniale», Ethnologie française, XXIX, 3, 1999, p. 333-336 (numéro spécial Musée, Nation : après les colonies). M. Adams Kathleen, «Identités ethniques, régionales et nationales dans les musées indonésiens", Ibid., p. 355-364.

Lee Wei-i : Doctorant en histoire et civilisations à l'École des hautes études en sciences sociales. 
L'étude de l'histoire des musées à Taiwan révèle des phénomènes similaires et complexes. Dans un contexte historique riche en revirements - la colonisation japonaise, la guerre civile entre nationalistes et communistes chinois, la guerre froide, l'autoritarisme, la modernisation, la démocratisation, la mondialisation - et sous la pression des tensions permanentes avec le continent et de l'isolement international, la construction de la symbolique culturelle des musées reflète le caractère ambigu et fluctuant de l'institution politique à Taiwan et la division de l'identité nationale.

Cet article vise à retracer les faits marquants de cette construction muséale centenaire en tentant d'en prendre en compte les dimensions essentielles : l'institutionnalisation, la constitution des collections, la symbolique architecturale, les activités d'exposition, le savoir et le discours scientifique, la délimitation de l'imaginaire... À travers ce réexamen global du processus d'institutionnalisation de la représentation culturelle et de son évolution à Taiwan, nous espérons parvenir à cerner, pour l'aire spécifique qu'est l'Asie extrême-orientale, comment s'articule la relation entre l'imaginaire culturel et le nationalisme dans l'histoire, plus précisément dans la transformation d'un régime autoritaire vers un régime démocratique.

Jusqu'au XviI ${ }^{e}$ siècle, l'île de Taiwan n'avait connu aucune forme politique de pouvoir centralisé, et consistait en un ensemble de communautés tribales formé de différents groupes ethnolinguistiques austronésiens. Pour s'assurer une plus grande maîtrise de leur commerce avec l'Asie du Sud-Est, la Chine et le Japon, les Pays-Bas conquirent le Sud de l'île en 1624, suivis par l'Espagne qui prit le contrôle du Nord en $1626^{4}$. Mais en 1662, l'armée du général Cheng Ch'eng-kung ${ }^{5}$ (16241662) s'empara à son tour de l'île après avoir défait les Hollandais. Commencèrent alors les immigrations massives des $\operatorname{Han}^{6}$ en provenance des provinces côtières du Fujian et du Guangdong, Han qui devinrent rapidement la population majoritaire sur l'île. En 1684, l'Empire des Qing vainquit le Royaume des Cheng, et l'île fut intégrée au territoire impérial. À la fin du XIX ${ }^{e}$ siècle, à la suite de la défaite de l'Empire des Qing dans la guerre sino-japonaise de 1894, Taiwan fut cédée au gouvernement du Japon l'année suivante par le traité de Shimonoseki et devint une colonie de l'Empire du Soleil levant.

4 - En 1642, les Pays-Bas vainquirent l'Espagne et prirent totalement le contrôle de la partie occidentale de l'île.

5 - L'essor de la famille des Cheng remonte à un célèbre pirate, Cheng Chih-lung (1604-1661), un Chinois originaire de la province du Fujian. L'influence de son armada s'étendait depuis le début du $\mathrm{XVII}^{e}$ siècle sur un vaste territoire maritime allant de Nagasaki au Japon jusqu'en Asie du Sud-Est, en passant par la ville de Xiamen en Chine. Ses entreprises furent continuées par son fils Cheng Ch'engkung dont la mère, Tagawa Matsu (1601-1646) était une Japonaise originaire d'Hirado à Nagasaki. Après l'écroulement de l'Empire des Ming en 1644 à Pékin, le jeune Cheng Ch'eng-kung, nommé général par la cour en exil des Ming du Sud, leva une puissante armée maritime à Xiamen en 1646 pour s'opposer en résistant contre le nouvel Empire Qing des Manchou. Son armée gagna en 1662 la guerre contre les Hollandais, et prenant pour base l'île de Taiwan, son fils put continuer à diriger les entreprises économiques et politiques de la famille jusque sa défaite contre les Qing. Voir Frank J. Tang, Dahanghai shidai de Taiwan [Taiwan in the age of exploration], Taipei, Guoshi, 2001.

6 - Han, l'ethnie dominante en Chine, représente $90 \%$ de la population chinoise. 


\section{La création du Musée du gouverneur de Taiwan (1908) : l'imaginaire colonial dans l'expansion de l'Empire japonais}

Le système muséal moderne fut introduit à Taiwan durant cette période de colonisation japonaise. En 1899, fut créé à Taipei au département des Propriétés coloniales (équivalent d'un ministère de l'Économie) un premier musée. Ce n'était qu'une salle d'exposition des marchandises; le même département fit ensuite installer un stand consacré à Taiwan lors de l'Exposition de l'industrie domestique à Osaka en 1903 puis à Tokyo en 1907, pour présenter la richesse de la colonie. L'année suivante, lors de l'inauguration de l'axe ferroviaire nord-sud, le gouvernement colonial annonça son intention d'organiser sur l'île même une grande exposition, afin de présenter de manière globale les réussites de sa gestion. Ce projet finit par aboutir à la création d'un grand musée polyvalent - le Musée du gouverneur de Taiwan.

Durant les premières années de la colonisation, les lourdes dépenses générées par les opérations militaires avaient suscité de vifs débats au sein du gouvernement à Tokyo : convenait-il de continuer à coloniser Taiwan, ou plutôt de l'abandonner? Il fut même question en 1897, lors d'une session parlementaire impériale, de la céder à la France contre cent millions de yens ou de la rétrocéder à la Chine. Ce n'est qu'à partir de 1907, quand l'île put apporter sa contribution à la métropole avec les taxes sur le sucre et les taxes douanières, que s'affirma à Tokyo la nécessité de garder Taiwan à titre de colonie. Dans ce contexte, le gouvernement japonais entendait bien, en construisant les premiers musées à Taiwan, démontrer les potentialités de l'exploitation de lîle en diffusant l'image de la richesse de la colonie. En retour, cette image de Taiwan construite par les musées pour la métropole, assurait aussi la légitimité politique du gouvernement colonial auprès des Taiwanais mêmes.

Le projet architectural du Musée du gouverneur illustre cette double démarche. En 1915, le musée quitta les locaux de la Loterie qu'il occupait pour son nouveau site, un bâtiment commémoratif dédié à Kotama Gentaro (1852-1906) et à Goto Shimpei (1857-1929), respectivement le gouverneur et l'administrateur des affaires civiles (Premier ministre) de la colonie entre 1898 et 1906. Pour rendre hommage à la «réussite» de la politique coloniale qu'ils menèrent, le nouvel administrateur des affaires civiles, Hiroya, lança en 1906 une collecte de fonds destinés à la construction d'un mémorial en leur honneur. Commencés en 1913, les travaux furent achevés en 1915. Le site choisi pour ce nouveau bâtiment était un temple de la Reine céleste, où l'on vénérait la déesse de la mer Matsu, qui était, et est encore, la divinité la plus importante pour les Chinois de Taiwan. L'architecte en charge du projet, Nomura Ichiro, issu de la première génération d'architectes formés à l'architecture occidentale à l'université impériale de Tokyo, adopta le style néoclassique avec en façade six colonnes doriques et une toiture en dôme. Le site symbolique de la religion traditionnelle des Chinois dans la vieille ville de Taipei se transforma en un musée moderne clairement occidental.

Plus que l'institutionnalisation administrative et le symbole architectural, l'élément fondamental dans cette construction de l'imaginaire muséal fut la constitution des collections et au-delà, leur interprétation. Dans cet exercice, le musée ne put que 
s'appuyer sur le développement du savoir scientifique de son temps. Dès 1897, deux ans après sa prise de pouvoir sur l'île, le gouvernement colonial avait achevé un premier recensement de la population et, en 1898, il avait mené une minutieuse enquête sur le territoire, suivie en 1905 par une enquête sur les plantes utiles de l'île. Parallèlement une trentaine de rapports d'enquêtes diverses sur les us et les coutumes, les systèmes sociaux, les données économiques, le droit civil, les lois administratives des Qing en vigueur à Taiwan furent rédigés entre 1901 et 1914 ${ }^{7}$. Tout le discours scientifique qui sous-tendait les activités du Musée du gouverneur se fondait sur ces rapports, et sa collection provint directement des collectes d'échantillons effectuées lors de ces enquêtes. Quant aux chercheurs, essentiellement en sciences naturelles, ils étaient pour la plupart formés à l'université impériale de Tokyo ${ }^{8}$.

Avec l'évolution de la politique expansionniste du Japon dans les années 1920, le Musée du gouverneur, en raison de sa localisation privilégiée, devint un des centres de la construction de l'imaginaire japonais sur l'Asie du Sud-Est. La collection du musée fut en effet élargie, des objets provenant de la Chine du Sud et de l'Asie du Sud-Est étant ajoutés à ceux collectés sur l'île. Après la création en 1928 de l'université impériale de Taipei, le développement universitaire des disciplines scientifiques dans des domaines aussi divers que l'ethnologie, l'anthropologie, l'archéologie préhistorique, la botanique tropicale, l'entomologie, la géologie ou encore la zoologie, vint renforcer les capacités de recherche scientifique du musée: nombre de ses conservateurs étaient en effet professeurs à l'université. À la fin de la colonisation japonaise en 1945, les collections du musée comportaient, selon l'inventaire établi par le gouvernement républicain, plus de 7000 pièces dont de nombreux objets provenant des aborigènes insulaires, des peuples de l'Asie du Sud-Est et de la Chine du Sud, mais aussi d'importants spécimens zoologiques, botaniques et géologiques collectés durant les enquêtes.

Mais la construction de la symbolique culturelle dans le musée peut être appelée à assumer bien plus ouvertement la tâche de consolidation de la légitimité politique. L'évocation de quelques grandes expositions organisées dans ce musée le montre très clairement. En 1916 fut d'abord organisée l'Exposition pour le développement commun de l'industrie, commémorant le $20^{e}$ anniversaire de la nouvelle ère politique, puisque c'est sous ce vocable que la colonisation y fut présentée. En 1933, l'Exposition commémorative en honneur du gouverneur Kotama et de l'administrateur des affaires civiles Goto fut présentée à l'occasion du $25^{\mathrm{e}}$ anniversaire de la fondation du musée. En 1935, l'Exposition commémorative pour le $40^{e}$ anniversaire de la nouvelle ère politique put accueillir plus d'un million de visiteurs ${ }^{9}$; enfin, en 1936, eut lieu l'Exposition commémorative du $30^{e}$ anniversaire de la mort du gouverneur Kodama où l'on revint

7 - Voir Ng Chiau-tong, Taiwan zongdufu [Le gouvernement colonial de Taiwan], Taipei, Qianwei, 2002, p. 81-97.

8 - Voir Ouyang Sheng-chih, Li Tzu-ning, «Bowuguan de yanjiu - yige lishi de huiyi» [Étude des musées - un souvenir de l'Histoire], in Numéro spécial pour le $90^{e}$ anniversaire du Musée provincial de Taiwan, 1999, p. 114-189.

9 - Lü Shaoli, Zhanshi Taiwan: Quanli, kongjian yu zhimin tongzhi de xingxiang biaoshu [Montrer Taiwan : représentation d'image du pouvoir, de l'espace et de la colonisation], Taipei, Maitian, 2005. 
encore aux figures fondatrices de la politique coloniale. Les expositions étaient devenues un rituel emblématique que le pouvoir colonial utilisait pour mener les campagnes exaltant sa légitimité politique.

À partir de la fin des années 1930, avec le déclenchement de la guerre, les expositions se transformèrent sensiblement en une machine de propagande pour la mobilisation militaire. Un simple survol des titres permet de prendre la mesure de cette évolution : Exposition des butins de guerre dans l'Événement de Chine ${ }^{10}$ en 1939; Exposition de l'histoire du développement des Japonais en Asie du Sud-Est depuis 2600 ans, organisée par le département de Mobilisation de l'esprit citoyen du gouvernement colonial en 1940; Exposition sur les Philippines; Exposition sur l'Asie du Sud-Est; Exposition sur les coutumes de l'ethnie Li de l'île de Hainan en 1942; Exposition sur la guerre pour la Grande Asie orientale en 1943. Ces expositions, et leur thématique militaire récurrente, tendaient à promouvoir auprès du public l'idée d'une légitimité de la guerre. Elles fournissaient également des informations sur les régions concernées, allant de Taiwan à la Chine du Sud, jusqu'en Asie du Sud-Est, afin d'étoffer et de "concrétiser» l'imaginaire du «Grand cercle de prospérité commune de l'Asie orientale».

\section{La création du Musée national d'histoire (1955): l'imaginaire historique de l'État-nation chinois}

À la fin de la seconde guerre mondiale, le gouvernement chinois de Nankin prit le contrôle de Taiwan avec la rétrocession de l'île par le Japon à la République de Chine (RdC). Moins de quatre années après son unification avec le continent, Taiwan en fut à nouveau séparée : dans la guerre civile qui opposait le Parti communiste et le Parti nationaliste (Kuomintang, KMT), le premier ayant pris le pouvoir et succédé au KMT à la tête de la Chine continentale, le gouvernement de Chiang Kai-shek (1887-1975) fut contraint de s'exiler à Taiwan en 1949, avec un million de réfugiés et d'importants transferts de patrimoine chinois et d'institutions républicaines sur l'île. Deux ans après, éclata la guerre de Corée. La force militaire des États-Unis intervint dans la sphère Asie-Pacifique. Sous la guerre froide, le gouvernement de Taipei préserva son siège au Conseil de sécurité de l'ONU en tant que représentant de la Chine. Et l'accord de défense mutuelle entre la République de Chine à Taiwan et les États-Unis, signé en 1954, ouvrit la voie à une deuxième vague d'institutionnalisation des grands musées à Taiwan. Mais cette fois-ci, Taipei n'était plus à la périphérie d'un État, mais bien au centre. Dans le processus d'installation de l'appareil d'État de la République de Chine à Taiwan, un musée consacré à l'histoire nationale fut créé dans la capitale «temporaire» de Taipei - un cas typique de construction de l'imaginaire national opérée au sein d'un État-nation moderne par le truchement de l'institutionnalisation muséale.

La Galerie nationale du patrimoine historique et artistique ouverte au public en 1956 fut rebaptisée Musée national d'histoire (MNH) par Chiang Kai-shek lors

10 - L'«Événement de Chine» est la formule faisant référence à l'attaque japonaise lancée le 7 juillet 1937 contre la ville de Wanping, qui provoqua l'incident du pont Marco Polo. À la suite de ces premières hostilités, l'armée japonaise envahit le 30 juillet Pékin et Tianjin, et le 13 août Shanghai. Ainsi fut déclenchée la guerre sino-japonaise. 
de sa visite l'année suivante. L'objectif affiché était de "renforcer l'éducation de l'esprit national, [et de] contribuer à la construction psychologique des citoyens». Le musée, d'abord installé dans l'ancienne salle d'exposition des marchandises, une construction en bois de 1899 de style japonais, fut reconstruit pendant plusieurs années pour devenir un bâtiment au style impérial chinois - tuiles de lazurites, murs rouges, et éléments de décoration classiques comme ce bas relief d'ornement incrusté sur les murs à l'entrée, représentant Confucius s'instruisant auprès de Laozi. L'ambition d'affirmer une identité chinoise était patente.

Le Musée national d'histoire pouvait en effet prétendre au titre de gardien de la haute civilisation chinoise, car il hérita, en sus des trésors restitués par le Japon après la guerre, d'une partie importante de l'ancienne collection du musée du Henan ${ }^{11}$, où furent conservés les objets recueillis lors des fouilles effectuées sur les vestiges de

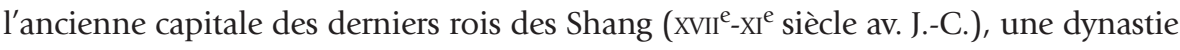
fondatrice de la civilisation chinoise. D'abord transférées dans l'arrière-pays par mesure de protection contre les risques de destruction japonaise, ces pièces furent ré-acheminées sur l'île en 1949, puis confiées au MNH en 1955 pour former la base de ses collections. Bien qu'il ait été créé pour répondre aux besoins politiques d'une identification historique en crise, le Musée national d'histoire n'en fut pas moins le dépositaire des précieuses reliques attestant de la grandeur de la civilisation antique de la Chine. Il témoignait aussi du développement de l'archéologie chinoise, rendu possible à partir des années 1920, par le retour au pays d'éminents chercheurs formés à l'étranger comme Li Chi ${ }^{12}$ et Fu Ssu-nien ${ }^{13}$, qui avaient su initier les recherches et préserver les trésors découverts au fil des longues années de troubles et de guerre.

11 - Pour retracer brièvement l'histoire de ce musée: à l'automne 1923, dans le district de Xinzheng au Henan fut mise au jour une centaine de pièces en bronze de la période Printemps et Automne (VIII - $\mathrm{V}^{e}$ siècle av. J.-C.) ; un comité de conservation du patrimoine antique fut créé sur place, comité transformé en musée ethnologique en 1928 par le gouvernement provincial. D'octobre 1929 à février 1930, des fouilles supplémentaires furent menées à deux reprises sur le site de la nécropole royale à Xiaotuncun, mettant au jour 3656 pièces de carapaces divinatoires et des objets en bronze datant des Zhou. Avec l'enrichissement de la collection, le musée ethnologique fut rebaptisé en 1930 musée du Henan. Et les fouilles continuèrent jusqu'en 1937, l'année de l'éclatement de la guerre. Voir Pao Chun-peng, Zhongguo bowuguan shi [L'histoire des musées de la République de Chine], Taipei, Comité de rédaction de l'Encyclopédie de la Chine, 1964, p. 30-32.

12 - Li Chi (1896-1979), docteur en anthropologie de l'université Harvard avec sa thèse intitulée The Formation of Chinese People: An Anthropological Inquiry, soutenue en 1923, fut nommé directeur de la nouvelle section archéologie de l'Institut d'histoire et de philologie de l'Academia Sinica en 1929. De 1929 à 1936, il dirigea les travaux de fouilles sur les sites des Shang au Henan. En 1934, il fut nommé directeur du comité préparatoire du Musée central à Nankin. En 1938, il fut élu membre honoraire du Royal Anthropological Institute en Angleterre. Après la guerre sino-japonaise, il fut chargé de l'enquête et de la rétrocession des reliques culturelles pillées par l'armée japonaise. En 1948, il participa activement au transfert des trésors impériaux et archéologiques sur l'île. À partir de 1949, il dirigea le département d'anthropologie de l'université de Taiwan. De 1955 à 1972, il fut directeur de l'Institut d'histoire et de philologie de l'Academia Sinica.

13 - Fu Ssu-nien (1896-1950) fut un des leaders du Mouvement du 4 mai en 1919. Après des études à Londres et à Berlin de 1920 à 1926, il fut un membre fondateur et longtemps directeur de l'Institut d'histoire et de philologie, organisme acteur principal dans les découvertes archéologiques réalisées sur les vestiges de la dynastie des Shang. En 1945-1946, il fut président par intérim de l'université de Pékin. Après son arrivé à Taiwan, il présida l'université de Taiwan de 1949 jusqu'à sa mort. 
Un relevé des intitulés des premières expositions temporaires organisées par le $\mathrm{MNH}$ éclaire le regard qu'il porte sur l'histoire chinoise. En 1956, eurent lieu plusieurs expositions: Patrimoine de la Plaine centrale (Henan), Fresques de Dunhuang et manuscrits de sutras des Tang; l'année suivante, ce fut Peintures et calligraphies des Qing, Porcelaine des Ming; en 1959, l'exposition principale fut consacré au Patrimoine des Han et des Tang; en 1961 fut installée, après l'agrandissement du Musée, une nouvelle salle de recherche dédiée aux cultures paléolithiques, mésolithiques et néolithiques, ainsi qu'à la Haute Antiquité chinoise. Enfin, en 1965, une exposition fut consacrée à l'emblématique Homme de Pékin ${ }^{14}$. Le Musée national d'histoire, avec ses collections permanentes et ses expositions temporaires, mit en scène un imaginaire historique centré sur la civilisation fondatrice des Shang et des Zhou ( $\mathrm{xl}^{\mathrm{e}}-\mathrm{III}{ }^{\mathrm{e}}$ siècle av. J.-C.), qui remontait jusqu'aux temps néolithique et paléolithique immémoriaux pour redescendre vers les grands empires chinois allant des Han (II ${ }^{\mathrm{e}}$ siècle av. J.C.-III ${ }^{\mathrm{e}}$ siècle apr. J.-C.) et des Tang (VII $-\mathrm{X}^{e}$ siècle) jusqu'aux Ming ( $\mathrm{XIV}^{e}-\mathrm{XVII}^{\mathrm{e}}$ siècle) et aux Qing ( $\mathrm{XVII}^{\mathrm{e}}-\mathrm{XX}^{e}$ siècle).

Les activités du MNH ne se limitaient pas à une reconstruction romantique de l'histoire de la Chine antique, car le musée avait été fondé pour aider à la construction de l'histoire d'un État nationaliste moderne. La création en 1965 de la salle d'exposition de «l'histoire de la révolution nationale» marqua l'institutionnalisation d'une histoire étatique centrée sur le Parti nationaliste chinois. La même année, fut aussi ouverte au sein du Musée la salle des "portraits des grands hommes historiques", mettant en scène trois groupes de grands hommes de la nation ${ }^{15}$ : les grands empereurs de l'histoire chinoise, comme Fuxi, Yao, Tang des Shang, Wu des Zhou et les empereurs des Tang, des Song, des Yuan et des Ming ${ }^{16}$; les sages antiques comme Cangjie $^{17}$, Confucius, Mencius et une cinquantaine de philosophes des Han, des Tang et des Song; et les héros nationaux modernes du Kuomintang (KMT), comme Sun Yat-sen, Chiang Kai-shek, Lu Hao-dong, Huang Xing, Song Jiao-ren et les 72 martyrs de Huanghuagang ${ }^{18}$. L'organisation en ordre chronologique des trois

14 - En 1929, sur le site de Zhoukoudian à Pékin, quatorze pièces de la boîte crânienne d'un homme d'il y a environ 500000 ans furent mises au jour. Certaines pièces furent perdues dans les méandres des années de guerre. En 1965, le Musée d'histoire naturelle de New York fit don d'une réplique au $\mathrm{MNH}$, qui organisa une exposition pour l'occasion.

15 - Voir Pao Chun-peng, Guoli lishi bowuguan de chuangjian yu fazhan [La création et le développement du Musée national d'histoire], Taipei, Comité de rédaction de l'Encyclopédie de la Chine, 1970, p. 32

16 - Fuxi, empereur mythique que la tradition consacre comme le premier ancêtre de la nation chinoise, est selon la légende l'inventeur des hexagrammes; Yao est le premier des trois rois sages mythiques que la tradition, confucéenne en particulier, considère comme l'exemple même du gouvernement vertueux; Tang des Shang, vainqueur du dernier roi tyrannique de la dynastie Xia, est le véritable fondateur de la dynastie des Shang; le roi Wu - militaire - des Zhou, vainqueur du dernier roi tyrannique des Shang, est le fondateur de la dynastie des Zhou.

17 - Cangjie, historiographe mythique du temps de l'Empereur jaune, est l'inventeur, selon la légende, de l'écriture chinoise.

18 - Sun Yat-sen (1866-1925), Père fondateur de la République de Chine. Lu Hao-dong (18671895), créateur du drapeau nationaliste, exécuté en 1895 lors de l'insurrection de Canton, fut surnommé par Sun Yat-sen le premier martyr de la révolution républicaine dans l'histoire de la Chine. Huang Xing (1874-1916), un des protagonistes majeurs dans la révolution nationaliste, fut l'initiateur de plusieurs insurrections dont celle de Huanghuagang en 1910. Song Jiao-ren (1882-1913), activiste révolutionnaire dès la fin des Qing, devint le leader de la majorité en tant que secrétaire du Parti après la victoire écrasante des nationalistes lors des élections en 1913; il fut assassiné par les autorités de Pékin avant la session plénière de l'Assemblée nationale. 
groupes de personnages tendait visiblement à créer une généalogie des ancêtres constitutifs de la nation chinoise, présentés comme les référents de la communauté nationale du $\mathrm{xx}^{e}$ siècle. Les membres de cette nation ne se limitaient plus aux seuls citoyens de l'État-nation moderne : tous les sujets de tous les empires chinois passés se retrouvaient dans une communauté imaginaire, traversant le temps et l'espace.

\section{La création du Musée national du Palais (1965):} l'imaginaire impérial de la République chinoise

Entreprise globale de légitimation, la politique nationale des musées se devait de mettre en ouvre une dimension internationale, liée à la nécessité de spécifier et de glorifier l'image du pays parmi les nations. La création du troisième grand musée de Taipei - le Musée national du Palais (MNP) - est directement liée à la situation diplomatique internationale de Taiwan.

Le Musée national du Palais, dans la mesure où il fut le seul véritable musée «impérial », par sa localisation initiale et par la filiation de ses collections, est en lui-même une institution légendaire. Inauguré le jour de la fête nationale, le 10 octobre, en 1925, dans l'année qui suivit le départ de l'empereur déchu des Qing, Puyi ${ }^{19}$, le musée fut d'emblée le plus prestigieux et le plus important en collections de toute la Chine. Il conservait, sur le site même de l'ancien palais impérial (d'où son nom), l'inestimable collection d'œuvres d'art chinois accumulée au fils des temps par les différentes cours impériales depuis la dynastie des Song ( $\mathrm{x}^{\mathrm{e}}$-XIII ${ }^{\mathrm{e}}$ siècle). Après l'incident du 18 septembre $1931^{20}$ à Shenyang, qui marqua le début de l'agression japonaise en Chine du Nord et ouvrit la voie à la création du Manchoukuo (Mandchourie) avec comme souverain l'empereur déchu Puyi, le Nord de la Chine se trouva dans une situation d'incertitude grandissante. Aussi le gouvernement de Chiang Kaishek ordonna-t-il dès 1932 le transfert vers le Sud du pays d'une sélection d'objets de qualité, comprenant porcelaines, jades, peintures et calligraphies, bronzes, livres anciens et archives impériales du Musée national du Palais, mais aussi les trésors du Palais d'été de Pékin, et ceux des anciennes résidences impériales de Rehe (Jehol) et de Shenyang (Palais de Moukden) ${ }^{21}$, ainsi que certaines pièces historiques venant de l'ancienne Académie impériale ${ }^{22}$, soit un total de 19557 caisses $^{23}$.

19 - Après son abdication en 1911, Puyi continua à vivre dans la Cité interdite jusqu'en 1924.

20 - Dans la soirée du 18 septembre 1931, la destruction d'un tronçon de chemin de fer à proximité de la ville de Shenyang sous contrôle japonais, servit de prétexte aux Japonais pour attaquer la garnison chinoise de Beidaying et commencer leur progression dans le Nord-Est de la Chine.

21 - Après trois ans de la République, en 1914, le ministère de l'Intérieur créa l'Institut d'exposition des antiquités de Beiping, dans la Cité interdite, accueillant 200000 pièces de trésors du Palais de Moukden à Shenyang et aussi de la résidence d'été à Rehe. Voir Pao Chun-peng, Zhongguo bowuguan shi [L'histoire des musées de la République de Chine], Taipei, Comité de rédaction de l'Encyclopédie de la Chine, 1964, p. 25.

22 - Dès la fondation de la République, en 1912, le ministère de l'Éducation nationale installa dans les locaux de l'ancienne Académie impériale un comité préparatoire du Musée national d'histoire, accueillant plus de 50000 pièces de la collection de la dynastie des Qing, créant ainsi le premier musée national de l'ère républicaine. Ibid, p. 23.

23 - Voir Gugong qishi xingshuang [Soixante-dix ans du Musée national du Palais], Taipei, Musée national du Palais, 1995, p. 87-92. 
Ainsi commençait pour ce riche patrimoine une pérégrination de près de trente ans : en 1933, il fut transféré dans les concessions françaises et anglaises de Shanghai, puis, en 1936, il fut ré-acheminé à Nankin, la capitale de la République de Chine. En 1937, avec l'éclatement de la guerre sino-japonaise, ces collections impériales durent à nouveau être mises en lieu sûr, au Sichuan, après un long périple dangereux et difficile par trois voies différentes. Elles ne retournèrent à Nankin qu'en 1947, après la guerre. L'intensification de la guerre civile entre les communistes et les Kuomintang obligea ceux-ci à un nouveau transfert des trésors à partir de décembre 1948 sur l'île de Taiwan. Organisé d'urgence, le transport ne put être réalisé que pour 5518 caisses, soit un ensemble de 640000 pièces $^{24}$. Entreposées sous des abris antiaériens construits en 1952 à Beigou, dans les montagnes de Taichung au centre de Taiwan, ces collections ne purent gagner le site actuel à Taipei qu'en 1965, après la réouverture du Musée national du Palais.

Dès 1955, le ministre de l'Éducation nationale Chang Chi-yun (1901-1985) avait déjà exprimé son intention de créer le Musée national d'histoire avec le patrimoine de la collection impériale pour favoriser "une éducation historique des citoyens ${ }^{25}$ ", mais il ne reçut pas l'aval du Bureau d'administration commune, véritable gérant de la collection. Ce bureau ${ }^{26}$, bien que placé sous l'autorité du ministère de l'Éducation, entendait maintenir l'autonomie de son organisation, en raison très certainement de la position historique particulière qu'occupait la collection placée sous sa responsabilité. Avant sa dissolution en 1965 avec la création du nouveau Musée national du Palais, il se concentra essentiellement sur des opérations de coopération avec l'étranger destinées à gagner une reconnaissance internationale: en 1955, une demande de subvention fut formulée par le Bureau auprès de l'Asia Foundation basée aux États-Unis, afin de construire la salle d'exposition de Beigou à Taichung, pour répondre aux besoins des chercheurs internationaux et des visiteurs étrangers. Dans le même esprit en 1959, le Bureau vota le projet de la construction d'un nouveau bâtiment à Taipei.

Pendant cette période, les rares grandes expositions des trésors de la collection impériale eurent toujours lieu à l'étranger ${ }^{27}$ : en 1959, en association avec l'agence

24 - Les objets transférés à Taiwan représentaient $22 \%$ des caisses déplacées depuis Pékin vers le sud. Ainsi 5518 caisses arrivèrent-elles à Taiwan : 2972 provenaient du Musée du Palais, 848 du comité préparatoire du Musée central, 976 de l'Institut d'histoire et de philologie de l'Academia Sinica, 644 de la Bibliothèque nationale, 60 d'archives du ministère des Affaires étrangères, 18 de cartes impériales des Qing et des Ming, anciennement conservées à la bibliothèque de Pékin. Voir Soixante-dix ans du Musée national du Palais, op. cit., p. 152-158.

25 - Voir Wang Yu-qing, "Sishi xingshuang hua shibo» [Quarante ans du Musée d'histoire], in Guoli lishi bowuguan jianguan sishi zhounian jinian wenji [Recueil pour le quarantième anniversaire de la fondation du Musée national d'histoire], 1995, Taipei, Musée national d'histoire, p. 26.

26 - Les collections transférées à Taichung appartenaient à différentes institutions: le Musée national du Palais, le comité préparatoire du Musée central, la Bibliothèque centrale, la bibliothèque de Pékin et le Studio national de films éducatifs. En août 1949, un organisme de gestion commune fut créé par le ministère de l'Éducation pour répondre à la situation de la guerre. Il fut baptisé Bureau national d'administration commune des musées et des bibliothèques.

27 - Hormis une tournée de photos de cinq cents trésors organisée en 1952 par le ministère de l'Éducation nationale et une exposition conjointe d'un mois et demi avec le Musée national d'histoire pour la cinquantième fête nationale en 1961 à Taipei, où le caractère nationaliste des événements était prépondérant. 
de presse japonaise Asahi Shimbun, une exposition des photos des peintures célèbres du Palais eut lieu dans le magasin de Shirokiya à Tokyo et le magasin $\mathrm{d}^{\prime}$ Hankyu à Osaka ${ }^{28}$. De mai 1961 à juin 1962, une grande exposition fut envoyée en tournée dans cinq musées américains : la National Gallery of Art à Washington, le Metropolitan Museum of Art à New York, le Boston Museum of Fine Art, l'Art Institute de Chicago, le M. H. De Young Museum de San Francisco, le nombre total de visiteurs atteignant $465000^{29}$. En 1964, lors du tricentenaire de la ville de New York, la République de Chine fut invitée à y participer à l'Exposition universelle. Une salle chinoise fut construite à cette occasion avec la participation du Bureau, de l'Academia Sinica et du Musée national d'histoire ${ }^{30}$. Outre ces quelques expositions exceptionnelles et le travail de conservation des œuvres, le Bureau d'administration a également mené plusieurs entreprises de catalogage et d'édition durant ses années d'activité. En effet, dans le contexte politique international de la guerre froide, où la Chine populaire était fermée au public étranger, cet ensemble patrimonial d'art chinois d'une qualité exceptionnelle constituait une source majeure d'informations de première main pour différents organismes internationaux spécialisés dans la recherche ou dans l'édition en matière de culture et d'histoire chinoise : en 1960 par exemple, la société américaine Shira publia un ouvrage d'art, incluant une cinquantaine de peintures célèbres de la collection du palais. En 1963, le professeur James Cahill réalisa quelque six mille clichés des œuvres pour la Freer Gallery of Art de Washington. Une copie des clichés fut confiée à l'Administration commune ${ }^{31}$.

En 1965, furent achevés les travaux de construction du Musée national du Palais à Taipei, dont le bâtiment conçu par l'architecte Huang Pao-yü ${ }^{32}$ imite la Porte du Midi, entrée principale de la Cité interdite à Pékin. Une calligraphie de Chiang Kai-shek, réalisée lors l'inauguration le 12 novembre, date anniversaire de Sun Yat-sen, baptisa le nouveau bâtiment, "Musée de Sun Yat-sen, dédié à la mémoire du centenaire de la naissance du Père fondateur de la République». Le nom ainsi donné au bâtiment destiné à abriter la collection impériale entendait instaurer une filiation entre les anciens et les nouveaux détenteurs de ces trésors, et par là, entre la République en exil et l'ancien Empire chinois. En réalité, Taipei était alors bien menacé de perdre la reconnaissance diplomatique dont le régime jouissait à titre de représentant de la Chine dans le monde ${ }^{33}$. Alors même que le continent s'engageait avec la Révolution culturelle dans une violente phase iconoclaste en 1966, une campagne pour la Renaissance

28 - Voir Soixante-dix ans du Musée national du Palais, op. cit., p. 182.

29 - Ibid., p. 175-180.

30 - Ibid., p. 181.

31 - Ibid., p. 188.

32 - Huang Pao-yü, diplômé du département d'architecture de l'université centrale à Nankin, faisait partie de la première génération d'architectes formés à l'école moderne, dont le style fut fortement influencé par la vague néo-classique chinoise du début du xx siècle.

33 - En 1964, la République de Chine (RdC, Taiwan) a rompu ses relations diplomatiques avec la France, le Congo, le Centre Afrique et le Sénégal à la suite de la reconnaissance diplomatique par ces derniers de la République populaire de Chine (RPC) en raison du principe de la Chine unique. En 1966, des conflits armés éclatèrent entre la Chine populaire et l'Union soviétique, et la dissension entre les deux pays communistes fit évoluer la position américaine envers la Chine populaire. Dès 1967, bien que la motion sur l'intégration de la Chine populaire dans l'ONU eût été rejetée par 58 voix contre 45, la République de Chine encourait bel et bien le danger de perdre son statut de représentant de la Chine. 
de la culture chinoise fut initiée par Chiang Kai-shek au nom de la défense de la tradition en 1967, et le Musée national du Palais fut naturellement désigné comme la base de ce mouvement. En effet, la réouverture du MNP à Taipei, à elle seule, suffisait au gouvernement républicain pour se définir comme le détenteur effectif de la culture chinoise et, par là, pour témoigner devant le reste du monde de la légitimité de la République de Chine, si «chinoise» par rapport à l'image d'une Chine populaire destructrice de ses traditions. Par un jeu de miroir, les images culturelles contrastées des deux rives fonctionnaient comme deux forces antagonistes livrées dans une lutte politique sur le terrain international, au profit, temporairement, de l'île.

\section{La création des musées nationaux des sciences naturelles et des technologies industrielles (1978): \\ l'imaginaire scientifique de l'État moderne}

La lutte diplomatique prit un tour nouveau en 1971, quand la République populaire de Chine fit son entrée à l'ONU, au détriment de la RdC. Taiwan ne représentait plus la Chine et le gouvernement de Taipei dut rapidement faire face à l'isolement international $^{34}$. Son échec diplomatique reconfigura les défis auxquels devait faire face Taiwan dans sa construction du nationalisme chinois. Par ailleurs, des forces d'opposition se développèrent rapidement dans la société taiwanaise ${ }^{35}$, et les discours indépendantistes se firent entendre ${ }^{36}$. Cette nouvelle situation mit un terme à la logique nationaliste axée sur la civilisation historique chinoise dans le fonctionnement des musées. L'accent fut transposé sur l'essor économique que commençait à connaître le pays et une quatrième vague de construction des grands musées à Taiwan fut progressivement initiée.

Le gouvernement de Chiang Ching-kuo (1910-1988), fils et successeur de Chiang Kai-shek, confronté aux crises de légitimité politique induites par la nouvelle situation, se vit contraint à abandonner un autoritarisme dur au profit d'un autoritarisme souple. Après quelques années d'active politique de modernisation industrielle, Chiang Ching-kuo décida d'étendre la modernisation au domaine de la culture, jusqu'à en faire le socle d'une nouvelle identité nationale. En 1978, à peine neuf mois après sa prise de fonction en tant que chef d'État, son gouvernement adopta un vaste ensemble de projets de construction des grands musées, comprenant un musée des Sciences naturelles, un musée des Technologies industrielles et scientifiques, et un musée de la Mer (qui se scinda en deux en 1989 : Musée national de la biologie marine et Musée national des technologies marines).

Situé à Taichung, le Musée national des sciences naturelles, ouvert au public en 1987, affiche pour objectif d'«assurer au niveau national la collecte, la recherche, la

34 - La RdC (Taiwan) a rompu ses relations diplomatiques avec l'Italie en 1970, avec le Chili, le Koweït, l'Autriche, la Turquie, l'Iran, la Sierra Leone, la Belgique, le Pérou, le Liban, le Mexique, l'Équateur, en 1971, avec le Japon, la Nouvelle-Zélande, l'Australie, la Jamaïque en 1972, avec l'Espagne en 1973, avec la Malaisie, Le Venezuela, le Brésil, la Gambie en 1974, avec le Viêt-Nam, les Philippines, la Thaïlande en 1975, avec la République centrafricaine en 1976, avec les États-Unis en 1978.

35 - Voir Samia Ferhat-Dana, Le Dangwai et la démocratie à Taiwan, Paris, L'Harmattan, 1998.

36 - Voir Claude Geoffroy, Le mouvement indépendantiste Taiwanais, Paris, L'Harmattan, 1997. 
conservation et l'exposition des données et des échantillons des sciences naturelles, afin de promouvoir la science auprès du peuple» dans quatre domaines : la zoologie, la botanique, la géologie et l'anthropologie. Le Musée national des technologies industrielles et scientifiques, ouvert en 1997 dans le Sud à Kaohsiung - le premier port du pays et la plus grande ville d'industrie lourde (acier, chantier naval, pétrochimie, machinerie) depuis 1970 -, est consacré à l'évolution des technologies et des sciences appliquées. Ouvert en 1997, le Musée national de la biologie marine est situé à Checheng, sur la pointe méridionale de l'île où se trouvent 1700 mètres de récif corallien, et présente trois aquariums sur les biotopes marins : le récif corallien, les eaux de Taiwan et les eaux du monde. Le Musée national des technologies marines, situé à Chilung dans le Nord-Est de l'île, le deuxième port du pays, se propose de montrer les applications des sciences et des technologies marines, et d'analyser les interactions entre les activités humaines et le milieu marin. On aura noté l'importante dimension maritime de ce dispositif muséal, dont la nature scientifique constituait un trait caractéristique de l'époque dans la construction symbolique d'un État moderne.

Parallèlement, à partir des années 1980, les grandes villes se dotèrent de musées d'art moderne. En 1983, le premier musée d'art moderne - le musée municipal des BeauxArts de Taipei - vit le jour, suivi en 1988 par le musée provincial des Beaux-Arts de Taiwan à Taichung, au centre de l'île. Le musée municipal des Beaux-Arts de Kaohsiung, inauguré en 1994 vint compléter le dispositif en couvrant la partie sud du territoire.

Cependant, alors que le processus de modernisation de la société taiwanaise donnait corps à la construction "progressiste» de ces musées scientifiques et artistiques, apparut une force «contraire» : une sorte de « retour à la tradition » répondant certainement au choc de cette même modernisation. Mais le retour à la tradition des années 1980 n'était plus celui des années 1960. Il ne s'agissait plus de la construction d'une tradition "lointaine, abstraite et élitiste», mais d'une tradition "quotidienne, concrète et folklorique», provenant de la base de la société taiwanaise. En 1981, fut créé le Comité de la construction et du développement culturels (CCDC, l'équivalent du ministère de la Culture). Prenant comme modèle les expériences du «village folklorique coréen » et du "village Meiji » du Japon, le premier président du CCDC, Chen Chi-lu (1923-), anthropologue de formation, voulut promouvoir la construction de deux «Jardins d'arts folkloriques» à Kaohsiung et à Ilan, dans le Nord-Ouest de l'île. Puis, pour faire droit aux traditions de la société aborigène, il initia la construction de «villages ethnologiques» à Wulai de Taipei et à Hualian, dans l'Ouest de l'île. En 1987, un musée provincial, le «Parc culturel des Compatriotes montagnards» de Taiwan fut ouvert au public. Et en 1990 le gouvernement de la province mit en chantier un Parc de culture et d'histoire de Taiwan à Nantou - le centre de l'île et le chef-lieu du gouvernement de la province de Taiwan - comprenant un ensemble de trois musées : musée des Folklores taiwanais, musée de l'Origine chinoise de l'histoire taiwanaise et musée des Archives taiwanaises.

La période fut également marquée par la construction du Musée national de la culture préhistorique à Taitung, dans le Sud-Ouest de l'île, un musée consacré à l'histoire des aborigènes taiwanais. À la différence des autres projets de grands musées nationaux de l'époque, la création de ce musée, en raison des circonstances 
particulières qui l'ont rendue possible, relève d'un hasard des temps modernes. Repéré depuis longtemps par les archéologues, le site finalement découvert lors de la construction du tronçon sud-est du circuit ferroviaire de l'île, en 1980, se révéla être la plus grande nécropole de sarcophages en pierre dans la région pacifique et de l'Asie du Sud-Est. Des fouilles de sauvetage orchestrées par les chercheurs de l'université de Taiwan ont permis la mise au jour de plus de vingt mille objets néolithiques, datant de 3300 à 300 av. J.-C. Cette importante découverte a conduit dès 1983 à la création d'un musée qui est ouvert officiellement au public depuis 2002.

\section{La création des musées nationaux "taiwanais" (1992-): l'imaginaire historique et politique avec l'essor du nationalisme taiwanais}

Avec les vives polémiques sur l'identité chinoise et taiwainaise que connut la société dans les années 1980, le nationalisme chinois dut faire face à des défis encore plus cruciaux à l'intérieur de l'île, surtout, à partir de 1987, quand l'abrogation de la loi martiale mit un terme à un règne autoritaire de trente-huit ans. Dès 1989, le tout nouveau Parti démocrate progressiste (PDP), créé trois ans auparavant par une stratégie dite "d'encerclement du centre par les périphéries», gagna 6 des 21 municipalités lors des élections municipales. Dans ce contexte de mutation politique et de mouvements sociaux très actifs furent créées, en 1989, les Archives de Ilan, qui devinrent en octobre 1993 le musée d'Histoire de Ilan - le premier musée officiel «d'opposition» consacré à l'histoire locale de Taiwan - lors de la célébration du $197^{e}$ anniversaire des premières immigrations Han dans la région d'Ilan. À partir de l'histoire locale, ce nouveau musée construisit une identité historique régionale en rupture avec l'interprétation du grand récit de l'histoire chinoise homologuée par l'autorité centrale. En 1997, pour en célébrer le cinquantenaire, fut ouvert au public le mémorial municipal de l'«Événement du 28 février ${ }^{37}$ ", dont la construction avait été promue par la mairie de Taipei, alors dirigée par Chen Shui-bian, l'actuel président indépendantiste. En moins d'une décennie, près d'une centaine de musées locaux nouveaux virent le jour à Taiwan.

C'est au début des années 1990, quand s'achevait le processus de transformation démocratique de la société autoritaire de Taiwan, qu'apparut la cinquième vague de création des grands musées. En 1992, des élections législatives eurent lieu en vue d'une refonte complète de l'Assemblée nationale en un Conseil législatif. Mobilisant les nouvelles forces politiques démocratiques, Lee Teng-hui, le président successeur de Chiang Ching-kuo, fonda sa légitimité politique sur la construction active d'une identité fortement taiwanaise. Une nouvelle hiérarchie culturelle se mit en place à cet effet. En 1992, Lee ordonna la création du comité préparatoire du musée provincial

37 - Après la reprise du contrôle de Taiwan par le gouvernement du Kuomintang, de violents conflits se nouèrent entre les Taiwanais et le nouveau gouvernement, en raison d'un ensemble de facteurs historiques complexes. En 1947 éclata l'Incident du 28 février (dit « du 228 ») où de nombreux intellectuels, notables et gens du peuple taiwanais furent exécutés. Cet événement constitue dans le processus de démocratisation de Taiwan, le symbole de la violence autoritaire du régime de Chiang Kai-shek. Voir Chen Tsui-lien, Ererba shijian yanjiu [Études sur l'Événement du 28 février], thèse de l'Institut d'études politiques de l'université nationale de Taiwan, 1994. 
d'Histoire de Taiwan. Dans la même logique, trois autres institutions nationales centrées sur la culture taiwanaise furent mises en chantier : le Centre national des arts traditionnels en 1996, le Centre national de la conservation et de la recherche des patrimoines culturels et le Musée national de la littérature taiwanaise en 1997. En 1997, par un amendement à la Constitution, une réforme dite d' "essentialisation» des provinces fut votée à l'Assemblée ${ }^{38}$, et la fin du mandat du dernier gouverneur de la province de Taiwan l'année suivante marqua la disparition de cet échelon administratif sur l'île. Ainsi, le futur musée provincial d'Histoire de Taiwan fut promu au rang de musée national, tout comme le musée provincial de Taiwan (l'ancien Musée du gouverneur) qui devint le Musée national de Taiwan. Les musées nommément «taiwanais» devinrent un nouvel indicateur de la politique culturelle du pays.

En 2000, lors des élections présidentielles, le candidat du PDP Chen Shui-bian fut élu, et le Parti nationaliste céda pour la première fois le pouvoir à un parti d'opposition. La construction d'une mémoire historique des Taiwanais s'appuie dès lors plus encore sur les musées et sur leur symbolique culturelle. Ainsi le Musée national d'histoire de Taiwan (MNHT) est-il actuellement en construction sur le site même du débarquement de l'armée de Cheng Ch'eng-kung à l'assaut du château fort hollandais au XVII ${ }^{e}$ siècle, dans la ville de Tainan, un lieu hautement symbolique pour l'histoire de Taiwan puisque la ville a été la capitale des Hollandais, puis des Cheng, puis le chef-lieu de la préfecture sous les Qing jusqu'à la fin du XIX siècle. Consacré à l'Histoire de l'île à travers l'évolution complexe de ses relations intercommunautaires, son processus de modernisation et ses échanges avec le monde extérieur, le musée a clairement voulu souligner ses caractéristiques taiwanaises, en commençant par sélectionner en 2001, comme lauréat du concours international d'architecture pour l'aménagement du musée, le projet de l'architecte taiwanais Chien Hsueh-yi. Abandonnant le style «impérial» - somptueuses toitures de palais chinois et éléments de décoration classiques - couramment adopté depuis un demisiècle pour les grandes constructions officielles à Taiwan, le projet de Chien propose un corps de bâtiment qui reprend des éléments essentiels aux constructions traditionnelles proprement taiwanaises : les maisons en dalles de pierre des aborigènes d'une part, et les murs en briques rouges de l'habitat populaire hoklo de l'autre. L'ensemble est situé dans un jardin conçu selon des principes écologiques en vogue, reproduisant le paysage typique de terrain humide et des végétations indigènes.

Parallèlement, plusieurs grands musées nationaux sont appelés depuis 2000 à une remise en scène de l'histoire de l'île. Le Musée national de Taiwan en particulier (l'ancien Musée du Gouverneur), après quarante ans d'activités réduites, organisa

38 - Après le transfert du gouvernement nationaliste sur l'île en 1949, la République de Chine n'eut plus sous son contrôle que la province de Taiwan et les îles Quemoy et Matsu de la province du Fujian, mais elle continuait à prétendre à la souveraineté sur les trente-cinq provinces de toute la Chine. Il existait pour cela dans le pays quatre échelons administratifs comprenant : le gouvernement central, la province, la municipalité/district et le village. Mais avec les élections libres des maires de Taipei et de Kaohsiung, du préfet de la province de Taiwan en 1994, puis du président de la République en 1996, le préfet de la province se retrouvait en charge d'un territoire quasi identique à celui du président. Cette superposition administrative entraînant des complications politiques inévitables, une réforme administrative fut lancée par la nouvelle présidence. 
en 2001, en coopération avec le comité préparatoire du MNHT, l'étonnante exposition L'Histoire $d u$ drapeau du tigre jaune, qui retraçait la tragique histoire oubliée de la République démocratique de Taiwan (du 23 mai au 21 octobre 1895), fondée après la signature du traité de Shimonoseki par des personnalités taiwanaises refusant la cession de l'île au Japon. En 2003, l'exposition Les Français nous attaquent - Taiwan et la guerre entre les Qing et la France, organisée au Musée, présenta au public la conquête inaboutie par l'armée française du Nord de l'île et la création de la province de Taiwan par l'Empire des Qing. À l'été 2006, à nouveau en coopération avec le comité préparatoire du MNHT, une exposition intitulée Hermosa : Maritime Taiwan and Spain fut organisée au Musée, avec la participation des Archivo General de Indias de Séville et du Museo José Luis Bello y González du Mexique, pour rappeler au public, selon les dire des organisateurs, les liens historiques entre l'Espagne et Taiwan. En plus de ces manifestations qui deviennent régulières, une grande exposition exceptionnelle, intitulée Ilha Formosa: The Emergence of Taiwan on the World Scene in the 17th Century fut organisée en 2003 au Musée national du Palais, en partenariat avec trente-sept musées et institutions dans le monde comme le Historisch Museum de Rotterdam, pour retracer l'histoire des relations que Taiwan entretenait avec le monde à cette époque. Le point de vue général adopté dans toutes ces expositions tranchait avec la vision officielle imposée pendant un demi-siècle par les autorités, qui présentait Taiwan comme un territoire chinois n'ayant jamais eu d'autonomie propre et toujours prêt à combattre les envahisseurs étrangers pour défendre la mère patrie.

Les actions menées par le gouvernement du PDP dans le domaine des musées ne se limitent pas à une reconstruction de la mémoire historique, mais cherchent avant tout à construire une mémoire politique commune d'un État nationaliste taiwanais. Élu président de la République en 2000, Chen Shui-bian a promi trois musées nationaux sur la mémoire politique contemporaine taiwanaise : d'abord un Mémorial national des droits de l'homme en 2002; puis l'année suivante, un Musée national de la politique parlementaire à Taiwan, destiné à conserver les archives de l'ancien Parlement de la province de Taiwan et celles concernant le développement de la gestion locale autonome à Taiwan. Et le 28 février 2007, lors des cérémonies pour les commémorations du soixantième anniversaire de l'Événement, s'est ouvert au public un Mémorial national de l'Événement du 28 février, en présence du président. Parallèlement, deux parcs commémoratifs consacrés aux persécutions contre les droits de l'homme sous le régime autoritaire du Kuomintang ont été créés sur les sites symboliques que sont les anciennes prisons où furent détenus les activistes politiques : sur l'île de Lutao et à la prison de Jingmei à Taipei. En mars 2007, le gouvernement du PDP a voulu rebaptiser «Mémorial de la démocratie de Taiwan» le Mémorial de Chiang Kai-shek, un monument ouvert au public en 1980 et situé au coeur de la ville de Taipei. Ces appels hautement politiques à la création de nouveaux musées cherchent à remodeler la mémoire politique des Taiwanais en y excluant l'influence du Parti nationaliste chinois, pour lui substituer le vécu politique des partis dissidents et du PDP dans l'espoir de consolider une nouvelle identité politique taiwanaise. 
Dès sa prise de fonction en 2000, le président Chen Shui-bian nomma un nouveau directeur pour le Musée national du Palais, Tu Cheng-sheng, membre de l'Academia Sinica, initiateur principal de la réforme des manuels d'histoire et actuel ministre de l'Éducation. Immédiatement, la question de l'évolution politique de ce musée emblématique fut posée. Un "processus de dépolitisation» se résumant essentiellement à une dénationalisation chinoise du Musée fut ouvertement engagé. La stratégie adoptée dans cette transformation consiste en un processus de «mondialisation» de ce musée, en définissant les œuvres qui y sont conservées, non plus comme les chefd'œuvres de la nation chinoise, mais comme un patrimoine culturel mondial. En 2002, la direction du musée a lancé le projet d'une antenne du Musée du Palais à Chiai dans le Sud du pays. Cinq nouvelles salles d'exposition y seront construites, et l'ensemble sera consacré, au dire des initiateurs du projet, à la «redécouverte de l'Asie» à travers les arts asiatiques de l'écriture, du bouddhisme, du textile, de la céramique et de l'artisanat. L'objectif avoué est bien de substituer à l'identité chinoise toujours prépondérante de l'institution la construction d'un horizon asiatique. La sélection en 2004 du lauréat à l'appel d'offre international d'architecture n'en est que la plus patente concrétisation. En choisissant des matériaux locaux - marbre noir taiwanais pour le socle du bâtiment ou bois d'hinoki, propre à Taiwan, pour sa structure -, en concevant l'espace global comme un ensemble d'îlots rappelant la nature insulaire du pays, avec une double voûte pyramidale symbolisant les deux sommets de l'île, en dessinant des jardins inspirés des arts décoratifs musulman, bouddhique et japonais, l'architecte américain Antoine Predock a remporté la victoire contre des projets avant-gardistes comme celui de Daniel Libeskind.

Les initiatives prises par le Parti indépendantiste au pouvoir d'une dénationalisation chinoise du Musée du Palais et de la construction de nouveaux musées portés par le nationalisme taiwanais suscitèrent de vives critiques des partis d'opposition, toujours partisans d'un nationalisme chinois. Cette lutte culturelle s'infiltre d'ailleurs à tous les niveaux de la société. La reconfiguration des musées locaux, menée sur la base du nationalisme taiwanais, rencontra également des résistances de la communauté de "continentaux», surtout de la seconde ou troisième génération, totalement intégrée dans la société taiwanaise. Après quelques décennies d'évolution, l'imaginaire nationaliste chinois, traditionnellement dominant dans cette communauté ${ }^{39}$, se voit remplacé par la construction d'une mémoire historique de l'exil même, laissant ainsi totalement ouverte la question de droit d'instauration à l'histoire sociale contemporaine de lîle.

Lors des élections municipales en 1998, le Parti nationaliste reprit le contrôle de la mairie de Taipei. Sous la direction du nouveau maire Ma Ying-jeou, le mémorial municipal de l'Événement du 28 février, jusqu'alors centré uniquement sur ce drame

39 - Voir Stéphane Corcuff, «Une identification nationale plurielle : les Waishengren et la transition identitaire à Taiwan, 1988-1997 », thèse de l'Institut d'études politiques de Paris, 2000. 
historique des "Taiwanais de souche», s'applique désormais à élargir ses thèmes d'exposition en y incluant les expériences historiques des immigrés dans la construction démocratique de l'île. En 2002, l'exposition sur les événements de Tiananmen de 1989 réintroduisit dans le champ d'exposition publique le problème de la politique chinoise. La gestion du Mémorial, parfois mouvementée, cristallise en effet les conflits entre les différents camps politiques. Les revendications de la communauté des immigrés dite des "Continentaux» se font également plus pressantes. En 2002, dans la municipalité de Hsinchu gérée par le Kuomintang, fut créé le premier musée du Village des familles d'anciens combattants, célébrant la mémoire des immigrés continentaux de 1949. La subvention attribuée par le Comité de la construction et du développement culturel (CCDC) du gouvernement indépendantiste suscita de vives critiques en raison de sa dénomination de "subvention pour des groupes sociaux fragiles ${ }^{40}$. On assiste ainsi à une diversification accrue dans la gestion des musées locaux, rendant leur relation avec l'autorité centrale de plus en plus complexe.

Parallèlement, les mouvements de revendication identitaire des Aborigènes connurent un rapide développement dans les années 1990. Au terme discriminatoire utilisé jusqu'alors de "Compatriotes montagnards" se substitua celui de "Aborigènes», la création d'un Comité ministériel des Aborigènes, en 1996, marquant la prise en compte de ces communautés dans les instances du gouvernement central. Un mouvement similaire chez les Hakka aboutit également en 2001 à la création du Comité ministériel des Hakka. Une structure d'identification communautaire multipolaire fut progressivement mise en place au sein même de l'institution politique, et commença à se mettre en scène dans les musées. Par exemple, en 1994 fut ouvert le premier grand musée privé consacré aux Aborigènes, le musée des Aborigènes Formosa de Shung Ye, situé juste en face du Musée national du Palais. En 2005, un premier musée public mono-ethnique des Aborigènes, le musée des Atayal à Wulai vit le jour. Sous les auspices du CCDC, furent construits trois musées de patrimoine Hakka à Kaohsiung, à Meinung et à Lioutui. Et après la création du Comité ministériel des Hakka en 2001, fut initié le projet de construction de cinq parcs culturels des Hakka à travers tout le pays. Le phénomène des identités multiethniques de l'île apparaît donc de plus en plus clairement dans le système muséal.

Face à ce phénomène de revendications identitaires multiethniques, le gouvernement du PDP tente de construire l'image d'une nation taiwanaise multiculturelle, en définissant la culture austronésienne des Aborigènes, tout comme la culture chinoise (des Hoklo, des Hakka et des Continentaux), comme une partie intégrante de la culture taiwanaise, jusqu'à en faire une marque de fabrique dans sa nouvelle politique de représentation diplomatique. Par exemple, au Centre culturel de Taiwan à Paris, eut lieu en 2002, avec la participation du Musée national de Taiwan, l'exposition Les Enfants de la mer - Reliques culturelles des Dawu, une exposition consacrée à la culture maritime du peuple austronésien de Dawu, habitant île de Lanyu près de la côte sud-est de Taiwan. L'année suivante, le même centre, avec le même musée, a organisé une exposition à nouveau consacrée à la culture aborigène de l'île, intitulée 
Les Aborigènes taiwanais et leurs dieux, en présentant une soixantaine de pièces liées au rituel religieux des populations aborigènes. L'image d'un peuple taiwanais aborigène et enfant de la mer laisse entrevoir celle que le gouvernement entend se donner dans l'espace international. C'est ainsi que le gouvernement taiwanais a présenté comme l'un des dix chantiers majeurs de construction de l'État, la création, à l'initiative du Musée national de la culture préhistorique à Taitung, d'un Parc naturel austronésien dédié à une culture qui couvre la très large zone des îles de Taiwan et de l'Asie du Sud-Est, mais aussi du Pacifique, de l'Océanie et de l'Océan indien. Ces opérations muséales indiquent clairement une préférence "austronésienne» de la part du gouvernement dans ses tentatives de redéfinition de la nation taiwanaise, en la rapprochant ainsi de l'Asie du Sud-Est et du Pacifique.

Dans cette mutation profonde des repères identitaires sur l'île, et en marge des mesures gouvernementales qui ne cessent d'opérer en faveur du nationalisme taiwanais depuis les années 1990, les deux grands musées historiques construits autour du nationalisme chinois entrent également dans une phase de redéfinition. S'appuyant sur la richesse de leurs propres collections, et à l'aide d'une gestion muséologique de plus en plus professionnelle, ils parviennent à un développement relativement autonome sur fond de mondialisation croissante, comme en témoignent les trois grandes expositions organisées ces dix dernières années dans des métropoles internationales par le Musée national du Palais. En 1996, après d'âpres négociations avec les autorités américaines qui ont abouti à un amendement des lois en vigueur pour garantir la sécurité diplomatique des ouvres, une exposition itinérante, plus de trente ans après celle des années 1960, intitulée Splendors of Imperial China, put circuler dans quatre musées américains: le Metropolitan Museum of Art à New York, l'Art Institute de Chicago, l'Asian Art Museum de San Francisco et la National Gallery of Art à Washington. À la suite du succès phénoménal de l'exposition, le Musée fut contacté par d'autres pays, européens notamment, et après des aménagements législatifs similaires à ceux des Américains, l'exposition Mémoire d'Empire fut organisée au Grand Palais à Paris en 1998 et, en 2003, l'exposition Schätze der Himmelssöhne (Trésors des fils du ciel) eut lieu à Berlin et à Bonn. Même si on ne peut nier le rôle de ces expositions dans la diplomatie culturelle d'un gouvernement taiwanais soucieux de rompre son isolement politique international, leur objectif affiché n'est plus l'exaltation nationaliste à la gloire de la civilisation chinoise, mais la mise en valeur esthétique des œuvres d'art du passé et leur interprétation plurielle dans des dynamiques historiques sans cesse renouvelées.

Cette évolution transparaît également à travers les grandes expositions organisées dans l'île même ces dernières années, au sein du Musée national du Palais et souvent en coopération avec des musées internationaux. Dans les années 1990, trois grandes expositions organisées en coopération avec des musées français attirèrent un nombre toujours croissant des visiteurs : en 1993, l'exposition Monet et les impressionnistes réalisée avec le musée Marmottan accueillit plus de 310000 visiteurs; en 1995, l'exposition Chef-d'œuvres du XVI-XIX siècle du Louvre fit près de 720000 entrées, et en 1998, l'exposition organisée avec le musée Picasso à Paris sur Chang Dai-Chien et Picasso - Art d'occident et d'orient atteignit 1,20 million d'entrées. À partir de la fin des années 1990, des collections venues de Chine furent prêtées pour être exposées 
au Musée national du Palais. En 1999, en coopération avec le musée de Sanxingdui au Sichuan, le MNP proposa au public l'exposition Les Mystères de Sanxingdui, suivie d'une exposition sur les reliques des tombes Han à Ma-wang-tui et d'une autre sur les tombes du roi de Nan-yueh, toutes deux faisant partie d'un programme d'exposition sur Art et culture de la dynastie des Han. Fort du succès de ses grandes expositions dans le monde, le MNP put bénéficier en retour de partenariats de plus en plus importants avec les musées étrangers pour offrir au public taiwanais des expositions de très grande envergure. En 2002, l'exposition intitulée De Poussin à Cézanne : 300 ans de peinture française rassemblait au MNP des œuvres provenant de vingt-sept musées français. En 2004, à la suite de l'exposition de Berlin et de Bonn, et en vertu de l'accord d'échanges signé avec les musées nationaux de Berlin, une exposition portant sur Un siècle de génie allemand : chef-d'œuvres du classicisme au modernisme a eu lieu à Taiwan. Enfin en 2007, le Musée accueille l'exposition 250 ans de collection au British Museum. Toutes ces expositions, qui dépassent le cadre de la collection impériale chinoise du Musée et incluent aussi bien les découvertes archéologiques récemment mises au jour en Chine que les œuvres d'art d'origine étrangère, marquent très clairement une réorientation d'ouverture impossible il y a encore peu.

Dans cette démarche d'ouverture, le Musée national d'histoire n'est pas en reste. Mettant en avant ses programmes de coopération internationale, il organisa lui aussi une série d'expositions centrées sur les grandes civilisations antiques du monde. Bénéficiant d'une excellente couverture médiatique, ces expositions au centre-ville de Taipei attirèrent à chaque fois facilement quelques centaines de milliers voire plus d'un million de visiteurs. En 1997, l'exposition en partenariat avec le musée d'Orsay et le musée des Beaux-arts de Kaohsiung, L'Âge d'or de l'impressionnisme, vit affluer plus d'un million de visiteurs. En 2000, à nouveau 1,05 million de personnes visitèrent l'exposition Armée de soldats en terre cuite - La culture des Qin, venue de Chine. Depuis, le Musée a proposé au public au rythme d'une exposition par an un parcours des grandes civilisations antiques. En 2001 avec le musée du Louvre, il organisa l'exposition L'Aurore de la civilisation: la Mésopotamie entre le Tigre et l'Euphrate, puis en 2002, avec le Musée national d'ethnologie et d'archéologie du Guatemala, une exposition sur Maya - Mystères dans la jungle. En 2003, le MNH accueillit une exposition intitulée Terre d'esprit et de mystère - Aperçu sur la civilisation indienne, organisée en coopération avec l'Indian Museum de Kolkata, et en 2004, le cycle se termina sur une exposition intitulée Visions d'Afrique - Les chef-d'œuvres de l'art primitif, grâce à la participation du musée Dapper, du Musée royal de l'Afrique Centrale en Belgique, et du musée d'Ethnologie de Vienne. Toutes ces expositions eurent en commun une nette ouverture sur le monde, qui par une sorte de dialogue implicite avec les collections permanentes du musée, désenclave ce dernier, qui échappe à sa position ambiguë et de plus en plus intenable de musée d'histoire chinoise, pour devenir un musée des civilisations.

Par ailleurs, d'autres musées comme le musée municipal des Beaux-Arts de Taipei et celui de Kaohsiung se sont également engagés dans d'importants projets d'échanges avec des musées internationaux, en particulier avec des musées français. Ainsi en 1991, l'exposition Le monde de Miró fut organisée au musée municipal des Beaux-Arts de Taipei en coopération avec la Fondation Maeght; en 1993, l'exposition Sculptures de Rodin organisée avec le musée Rodin de Paris accueillit quelque 250000 visiteurs 
L'APPARITION DES DIFFÉRENTS MUSÉES DE TAIWAN ET LEUR LOCALISATION

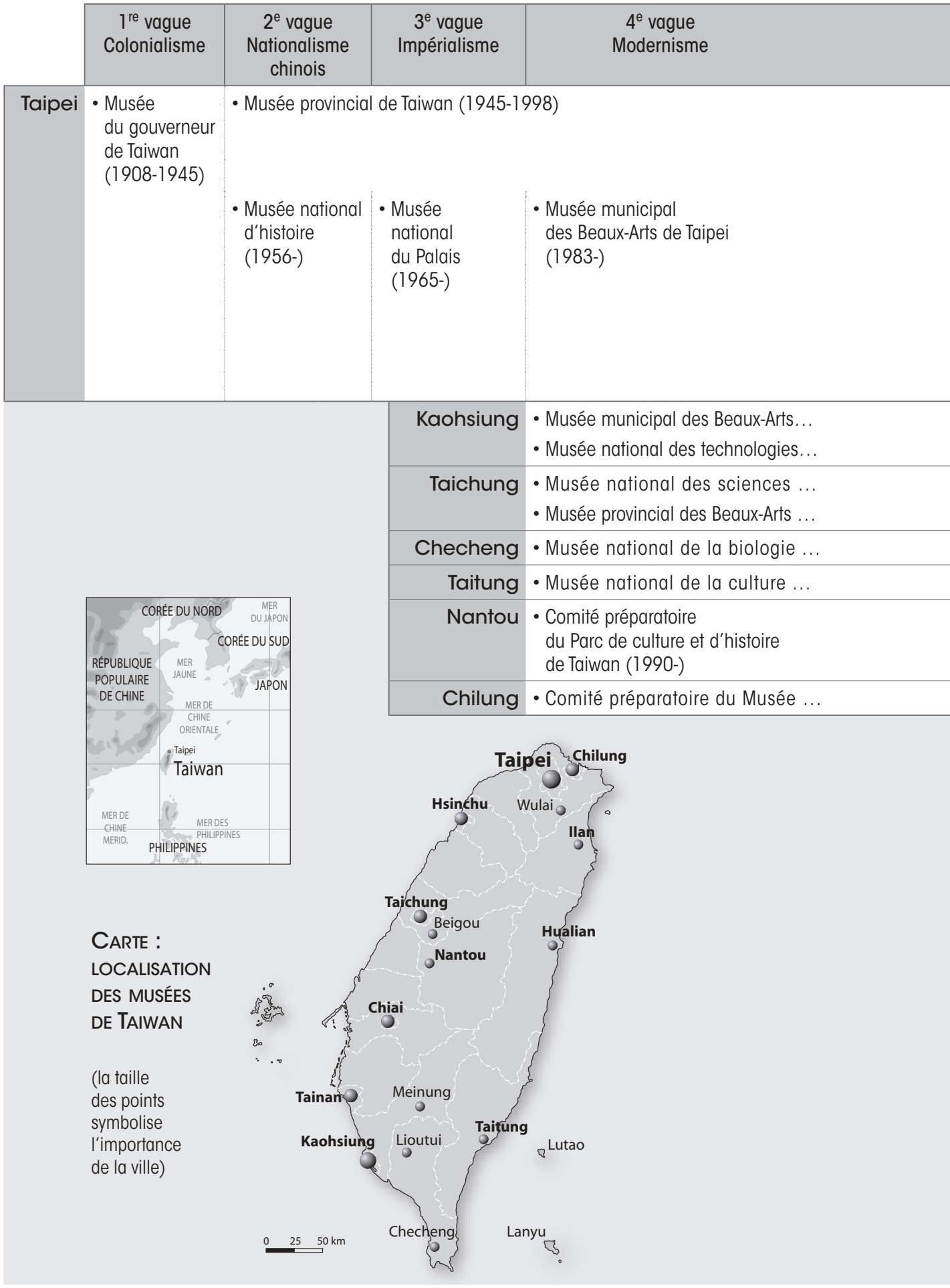




\section{$5^{\mathrm{e}}$ vague \\ Nationalisme taiwanais}

- Musée national de Taiwan (1998-)

- Mémorial municipal de l'Événement du 28 février (1997-)

- Mémorial national des droits de l'homme (2002-)

- Musée des Aborigènes Formosa de Shung Ye (1994-)

- Comité préparatoire du Mémorial national

de l'Événement du 28 février (2007-)

- Comité préparatoire du Parc mémorial

de la loi martiale de Jingmei (2007-)

... de Kaohsiung (1994-)

... industrielles et scientifiques (1997-)

- Musée de patrimoine Hakka de Kaohsiung (1998-)

... naturelles (1987-)

...de Taiwan (1988-1998), musée national depuis 1998

... marine (1997-)

... préhistorique (2002-)

- Musée national de la politique parlementaire à Taiwan (2003-)

... national des technologies marines (1997-)

Tainan • Comité préparatoire du Musée provincial d’histoire de Taiwan (1992-1998), musée national depuis 1998

- Comité préparatoire du Centre national de la conservation et de la recherche des patrimoines culturels (1997-)

- Comité préparatoire du Musée national de la littérature taiwanaise (1997-)

Ilan • Musée d'Histoire de llan(1993-)

- Comité préparatoire du Centre national des arts traditionnels (1996-)

Lutao • Comité préparatoire du Parc mémorial des droits de l'Homme de Lutao (2001-)

\begin{tabular}{|c|c|c|}
\hline & Chiai & $\begin{array}{l}\text { - Comité préparatoire de l'Antenne } \\
\text { du Musée national du Palais (2002-) }\end{array}$ \\
\hline & Hsinchu & $\begin{array}{l}\text { - Musée du Village des familles } \\
\text { d'anciens combattants } \\
\text { de Hsinchu (2002-) }\end{array}$ \\
\hline & Lioutui & $\begin{array}{l}\text { - Musée de patrimoine Hakka } \\
\text { de Lioutui (2001-) }\end{array}$ \\
\hline & Meinung & $\begin{array}{l}\text { - Musée de patrimoine Hakka } \\
\text { de Meinung (2001-) }\end{array}$ \\
\hline (c) 2007 Lee \& Song & Wulai & - Musée des Atayal (2005-) \\
\hline
\end{tabular}


à Taipei; et en 1999, l'exposition Chef-d'œuvres du musée de l'Orangerie reçut la visite de 630000 personnes à Taipei et à Kaohsiung. Le succès populaire de ces expositions montre bien à quel point les musées à Taiwan, surtout les musées d'art, ont été intégrés depuis les années 1990 dans des mouvements culturels mondialisés; l'imaginaire multiculturel des civilisations du monde et sans doute un consumérisme culturel propres aux grandes métropoles y jouent un rôle de plus en plus prépondérant.

\section{L'imaginaire des musées au-delà de l'identité?}

Deux points de vue sont portés sur les tensions, voire les conflits, générés par une identité nationale divisée et par les revendications multiculturalistes exprimées dans les musées à Taiwan. L'un est plutôt pessimiste. Il considère que le nationalisme domine toujours profondément les choix collectifs de l'île, et que, dans les conflits politiques entre les différentes communautés, il revient toujours aux vainqueurs le droit d'instaurer leur hégémonie culturelle : celle des Japonais, puis celle des nationalistes chinois du KMT, et maintenant celle des nationalistes taiwanais du PDP. Le deuxième point de vue, plutôt optimiste, voit dans l'essor de la nouvelle identité taiwanaise une mise en valeur concrète d'un multiculturalisme, qui modifierait les rapports intercommunautaires sur l'île, renonçant désormais à la quête d'un imaginaire national homogène traditionnel. Les deux perspectives nous semblent justes dans la mesure où elles reflètent effectivement une certaine évolution globale de la société, que l'on peut aussi observer dans les musées taiwanais. Mais elles échouent toutes deux à rendre compte d'un fait majeur spécifique à Taiwan, à savoir l'assimilation identitaire de moins en moins possible.

Les expériences traumatisantes de l'autoritarisme colonial japonais et du nationalisme chinois ont engendré une revendication identitaire taiwanaise naturellement tentée par un nationalisme spécifique; mais la position particulière de l'île dans le contexte international lui interdit tout espoir de reconnaissance officielle, non seulement à cause des menaces venant de Pékin, mais aussi en raison des intérêts géopolitiques particuliers des puissances occidentales, en particulier des Américains. Aussi, mis à part quelques irréductibles indépendantistes confirmés, la plupart des Taiwanais gardent une attitude plutôt réservée vis-à-vis du nationalisme taiwanais, voire de la logique même du nationalisme. Les manœuvres ouvertement nationalistes du gouvernement du PDP dans la promotion des musées proprement «taiwanais» suscitent en effet de moins en moins de ferveur : l'attention accordée à la création du Mémorial national du 28 février a été bien moindre, en envergure et en profondeur, en 2007 qu'en 1997 (mémorial municipal de Taipei); comme si la symbolique politique, surjouée par les autorités, avait perdu de son efficacité.

Les initiatives muséographiques multiculturalistes ont également très vite rencontré des impasses, après les premiers moments d'émotions liés à la reconnaissance des identités communautaires jusque-là négligées, voire opprimées. Poussés par des besoins politiques souvent liés aux impératifs électoraux, les projets se sont multipliés tout en manquant de consistance. Mais le plus grand obstacle que rencontrent ces projets en tant que constructions muséales reste la logique même de leur fonctionnement, qui se résume pour l'essentiel en une mobilisation identitaire, 
dépourvue de l'ouverture nécessaire à la vie de l'institution culturelle qu'est un musée. Qui plus est, dans une société qui connaît de plus en plus la pression de l'individualisme d'un côté, et qui pratique un métissage communautaire de l'autre, l'appel à des identités collectives sans réel impact sur la vie quotidienne des gens reste évidement toute symbolique. La vague de construction des musées ethniques sous la bannière du multiculturalisme n'a donné pour le moment que trop de «musées à moustiques», comme on le regrette souvent à Taiwan.

Pour répondre aux défis que représente le développement des musées à Taiwan, bien plus que de déterminer quel sort sera réservé au «nationalisme» ou au «multiculturalisme», il importe avant tout de comprendre et de respecter les possibilités propres à l'institution culturelle que constitue un musée. Les établissements qui attirent le plus de visiteurs ont tous adopté une telle démarche. Le Musée national des sciences naturelles par exemple est, depuis sa création dans les années 1980, le musée le plus visité à Taiwan. En plus d'une collaboration régulière avec le milieu de l'enseignements primaire et secondaire qui lui assure un nombre important de visiteurs, l'aspect ludique associé à la recherche et les coopérations actives avec la société civile que le musée met en place depuis vingt ans sont sans doute les clés de son succès auprès du public. L'évolution récente du Musée national du Palais et du Musée national d'histoire en offre d'autres exemples, sans doute plus éclairants encore. Ces deux grands musées, les plus marqués dans le passé par leur appartenance identitaire, ne peuvent ni continuer de jouer de l'imaginaire nationaliste chinois, ni embrasser le nationalisme taiwanais naissant. Confrontés aux impératifs nouveaux de la mondialisation, ils se sont finalement détachés de la logique identitaire politique en mettant en œuvre une démarche culturelle d'ouverture sur le monde. Les expositions temporaires qu'ils ont accueillies ou exportées, en mettant l'accent sur les accomplissements civilisationnels ou artistiques, déjouent les habitudes d'identification entre le patrimoine et la nation, et restituent à leurs collections leur nature d'œuvres d'art ou de témoins des civilisations humaines, en dépassant les frontières nationales traditionnelles. Si l'on peut soupçonner cette reconversion de participer à une nouvelle culture de consommation mondialisée, on ne peut nier que cette démarche portée par une logique des échanges contribue à l'ouverture et à l'enrichissement de notre imaginaire, qui reste sans doute, ou devrait toujours rester, la mission véritable d'un musée. 